\title{
Flutuação populacional do ácaro-da-ferrugem-da-videira em vinhedo comercial em Candiota, RS, com diferentes métodos de amostragem
}

\author{
Populational fluctuation of the grape rust mite in commercial vineyard in Candiota, Rio Grande do Sul \\ State, with different sampling methods
}

\author{
Paulo Ricardo Ebert Siqueira ${ }^{\mathrm{I}^{*}}$ Anderson Dionei Grützmacher ${ }^{\mathrm{II}}$ Marcos Botton ${ }^{\mathrm{II}}$ \\ Rosete Aparecida Gottinari Kohn ${ }^{\mathrm{I}}$
}

\section{RESUMO}

\begin{abstract}
Nos vinhedos da Região da Campanha do Rio Grande do Sul, o ácaro-da-ferrugem-da-videira, Calepitrimerus vitis (Nalepa, 1905) (Acari: Eriophyidae), tem sido encontrado com frequência desde a safra 2004/2005 associado com sintomas de bronzeamento nas folhas. A flutuação populacional de $\boldsymbol{C}$. vitis na cultivar 'Cabernet Sauvignon' foi estudada em vinhedo comercial localizado em Candiota, RS, durante as safras agrícolas 2007/2008 (de novembro a junho) e 2008/2009 (de outubro a maio). A amostragem foi realizada nas folhas e através de armadilhas constituídas por fitas adesivas de dupla face instaladas nos ramos de produção. O pico populacional, na primeira safra, ocorreu em março de 2008 quando foram registrados 0,34 indivíduos por $\mathrm{cm}^{2}$ da face abaxial das folhas medianas $e$ 29,48 indivíduos por armadilhas. Na segunda safra, o pico populacional foi menos intenso e ocorreu em outubro de 2008, quando foram detectados 0,11 indivíduos por $\mathrm{cm}^{2}$ da face abaxial das folhas medianas e 0,43 indivíduos por armadilhas. Foi detectado que o início do deslocamento de $\boldsymbol{C}$. vitis para os locais de hibernação ocorre no verão, a partir de fevereiro. As armadilhas adesivas foram mais eficientes para identificar a presença de $\boldsymbol{C}$. vitis no vinhedo do que a avaliação direta nas folhas. Uma correlação positiva foi encontrada entre o número de $\boldsymbol{C}$. vitis na face abaxial das folhas e o percentual de folhas com infestação.
\end{abstract}

Palavras-chave: Calepitrimerus vitis, monitoramento, Vitis vinifera.

\section{ABSTRACT}

In the vineyards of the Region of the Campanha of Rio Grande do Sul, Brazil, the grape rust mite Calepitrimerus vitis (Nalepa, 1905) (Acari: Eriophyidae), has been found frequently since the 2004/2005 harvest associated with symptoms of tanning on the leaves. The fluctuation of $\boldsymbol{C}$. vitis in 'Cabernet Sauvignon' was studied in a commercial vineyard located in Candiota, RS, during the agricultural years 2007/ 2008 (November-June) and 2008/2009 (October-May). The sampling was carried out on the leaves and by using traps constituted by double-faced adhesive installed in the branches of production. The populational peak in the first year occurred in March, 2008, which recorded 0.34 individuals per square centimeter of the abaxial face of the medium leaves and 29.48 individuals per traps. In the second year of the experiment, the populational peak was less intense and occurred on October, 2008, when it was detected 0.11 individuals per square centimeter of the medium leaves abaxial faces and 0.43 individuals per traps. It was detected that the beginning of the displacement of $\boldsymbol{C}$. vitis to hibernation places occurs on summer, starting in February. The adhesive traps were more efficient in identifying the presence of $\boldsymbol{C}$. vitis in the vineyard than the direct evaluation on leaves. A positive correlation was found between the number $\boldsymbol{C}$. vitis on the abaxial surface of leaves and percentage of leaves infested.

Key words: Calepitrimerus vitis, monitoring, Vitis vinifera.

\section{INTRODUÇÃO}

Calepitrimerus vitis (Nalepa) (Acari: Eriophyidae) é uma importante espécie fitófaga que ocorre na cultura da videira, causando bronzeamento e deformidade de folhas, encurtamento de ramos, atraso na brotação da primavera e morte das gemas

'Curso de Agronomia, Universidade da Região da Campanha (URCAMP), Avenida Tupy Silveira, 2099, 96400-110, Bagé, RS, Brasil. E-mail: siqagro@uol.com.br. *Autor para correspondência.

"Departamento de Fitossanidade, Universidade Federal de Pelotas (UFPel), Pelotas, RS, Brasil.

III Embrapa Uva e Vinho, Bento Gonçalves, RS, Brasil. 
(BERNARD et al., 2005). As perdas de produção decorrentes do ataque de $\boldsymbol{C}$. vitis apresentam variação com o local e o nível de infestação. Na Austrália, BERNARD et al. (2003) registraram perdas de até 13\%, enquanto nos Estados Unidos da América, WALTON et al. (2007) constataram até $24 \%$ de perdas na produção da videira, não havendo, até o momento, trabalhos de avaliação de perdas por $\boldsymbol{C}$. vitis no Brasil.

Os machos de $\boldsymbol{C}$. vitis são originários de partenogênese e ocorrem na primavera e no verão. Já as fêmeas são originárias de reprodução sexuada e podem ser do tipo primaveril ou protóginas ou, ainda, formas de inverno ou deutóginas, as quais surgem com a redução da temperatura a partir do outono como resposta das ninfas à senescência das folhas da videira (KEIFER, 1942; CARMONA \& DIAS, 1996; MORAES \& FLECHTMANN, 2008). C. vitis hiberna no interior das gemas ou sob o córtex nos ramos de produção ou no tronco e, na primavera, migra para as folhas, na metade basal dos ramos, onde se alimenta e oviposita (BERNARD et al., 2003).

Dentre as estratégias adotadas para o manejo de $\boldsymbol{C}$. vitis, predomina o controle químico, através da aplicação de acaricidas durante o inchamento das gemas, buscando reduzir a população de deutóginas no momento do deslocamento dos abrigos hibernais em direção às gemas em abertura na primavera. Nessas condições, a necessidade de controle é determinada pela intensidade do bronzeamento verificado nas folhas no verão e no outono anterior, com as reaplicações variando conforme a presença de C. vitis quando ocorrerem os estágios de 3 a 4 e, posteriormente, de 8 a 10 folhas totalmente expandidas (BERNARD et al., 2003).

Devido ao reduzido tamanho, o método empregado para a coleta e a detecção de $\boldsymbol{C}$. vitis apresenta papel decisivo tanto na pesquisa quanto em programas de controle (MONFREDA et al., 2010), sendo utilizados com frequência armadilhas adesivas (BERNARD et al., 2005; WALTON et al., 2007) ou a extração por lavagem (PÉREZ-MORENO \& MORAZAZORRILLA, 1998). Na Austrália, JAMES \& WHITNEY (1993) monitoraram a população de $\boldsymbol{C}$. vitis através de um sistema binomial de presença-ausência nas folhas e ramos, justificando o emprego desse método devido à dificuldade de localização e de contagem da espécie. De acordo com KLOCK (2008), nos vinhedos do Rio Grande do Sul infestados $\operatorname{com} \boldsymbol{C}$. vitis, essa foi a espécie de ácaro mais abundante, apresentando o pico populacional em janeiro de 2007.

Neste trabalho, foi avaliada a flutuação populacional de $\boldsymbol{C}$. vitis em vinhedo comercial da cultivar 'Cabernet Sauvignon’ na Região da Campanha do Rio Grande do Sul, por duas safras consecutivas, empregando-se como técnicas de amostragem armadilhas adesivas e a avaliação direta nas folhas.

\section{MATERIAL E MÉTODOS}

$\mathrm{O}$ experimento foi conduzido durante as safras agrícolas 2007/2008 e 2008/2009 no município de Candiota (31 23 '58,5'S, 5345'47'W e altitude de 256m), na Região da Campanha do Estado do Rio Grande do Sul, em vinhedo da cultivar 'Cabernet Sauvignon' enxertada sobre porta-enxerto SO4. O vinhedo foi implantado em 2002 em solo classificado como Luvissolo Háplico Órtico típico (STRECK et al., 2008), no sistema de espaldeira, com espaçamento de $3,0 \mathrm{~m}$ entre as fileiras e 1,0m entre as plantas, com histórico de infestação por $\boldsymbol{C}$. vitis.

A flutuação populacional de $\boldsymbol{C}$. vitis foi monitorada mediante o emprego de armadilhas adesivas e através da coleta de folhas. No início do experimento, uma amostra de ácaros foi encaminhada para identificação ao Dr. Noeli Juarez Ferla, taxonomista do Centro Universitário UNIVATES, Lajeado, RS, com a espécie confirmada como sendo $\boldsymbol{C}$. vitis. A área experimental consistiu de uma fração do vinhedo, formada por 120 fileiras, cada qual com 60 plantas. $\mathrm{O}$ delineamento adotado foi inteiramente casualizado, com a escolha de uma planta em uma das fileiras a cada data de amostragem. $\mathrm{O}$ monitoramento com armadilhas adesivas consistiu na adaptação da metodologia descrita por BERNARD et al. (2003), empregando um segmento de fita adesiva dupla face (Adelbras ${ }^{\circledR}$ ) com $11 \mathrm{~mm}$ de largura, incolor, posicionado no ramo de produção entre a primeira e segunda gema, de modo a circundar o ramo. Durante o ano agrícola 2007/2008, entre 17 de agosto e 03 de novembro de 2007, foram monitoradas semanalmente 25 plantas visando a detectar o movimento das formas hibernantes do tronco para as brotações. As armadilhas foram mantidas no vinhedo por 72 horas. Passado esse período, foram recolhidas, distendidas e afixadas em lâminas de vidro de microscopia, no mesmo sentido em que se encontravam na planta, e acondicionadas em estojos porta-lâmina. No laboratório, as lâminas foram analisadas sob microscópio estereoscópico com aumento de 60X. A partir de 9 de novembro de 2007, foi iniciado o monitoramento simultâneo de $\boldsymbol{C}$. vitis, nas mesmas plantas, com observação nas folhas, além das armadilhas adesivas. O intervalo de amostragem com armadilhas adesivas foi quinzenal, conforme sugerido por WALTON et al. (2007). O número de armadilhas foi ampliado para 50, em cada uma das 15 coletas, até 3 de 
junho de 2008. Em cada planta, foi amostrada uma folha do terço basal, uma do terço mediano, uma do terço apical e um broto apical do ramo de produção. As folhas foram analisadas também em microscópio estereoscópico, com aumento de 60X, contando-se o número de ácaros em um centímetro quadrado de cada folha, na porção central da face abaxial, adaptando a metodologia empregada por DUSO et al. (2004), enquanto o broto foi avaliado em toda a superfície.

Na safra agrícola 2008/2009, as coletas foram realizadas em 10 datas, entre 2 de outubro de 2008 e 5 de junho de 2009. Nesse período, foram sorteadas, em cada amostragem, 300 plantas para receberem as armadilhas adesivas, que foram instaladas e avaliadas de forma semelhante à safra anterior. Por ocasião do recolhimento das armadilhas foram coletadas, de cada planta, uma folha do terço mediano, por ter sido encontrado maior número de $\boldsymbol{C}$. vitis em folhas desta posição no primeiro ano de avaliação.

O cálculo da percentagem de incidência nas folhas e nas armadilhas adesivas foi baseado no sistema presença-ausência, sendo determinado, a cada data de avaliação, o número de armadilhas e de folhas de cada posição com a presença de $\boldsymbol{C}$. vitis, o qual foi dividido pelo número total de armadilhas ou folhas observadas.

Para detectar o início da atividade de deslocamento de $\boldsymbol{C}$. vitis dos ramos para o tronco, foram considerados, em movimento descendente, os espécimes capturados nas armadilhas adesivas com o gnatossoma orientado para o solo e, em movimento ascendente, no sentido tronco-ramo, os espécimes capturados com o idiossoma voltado para o solo. $\mathrm{O}$ percentual de indivíduos em movimento descendente foi calculado em relação ao total de capturas.

Os tratamentos fitossanitários durante a condução dos experimentos foram realizados conforme a decisão do produtor, de modo a não interferir nas condições normais de cultivo local, seguindo o adotado por DE LILLO et al. (2004). Houve aplicações de Kumulus DF (enxofre 800g i.a. $\mathrm{kg}^{-1}$ ) em 30 de novembro de 2007, na dosagem de $1.710 \mathrm{~g} \mathrm{ha}^{-1}$ e, em 20 de novembro de 2008, na dosagem de $2.627 \mathrm{~g} \mathrm{ha}^{-1}$, possuindo este produto ação acaricida sobre $\boldsymbol{C}$. vitis (BERNARD et al., 2003).

Foram obtidas informações meteorológicas na estação automática existente no vinhedo e realizada correlação entre o número de $\boldsymbol{C}$. vitis encontrados nas folhas medianas e nas armadilhas adesivas com a temperatura média diária e a precipitação pluviométrica no período de 7 e 14 dias antes das coletas.

\section{RESULTADOS E DISCUSSÃO}

Nas armadilhas adesivas utilizadas para detecção do início da atividade de $\boldsymbol{C}$. vitis após a hibernação, entre 17 de agosto a 3 de novembro de 2007, apenas um exemplar foi capturado, em 2 de outubro. Este deslocamento foi mais tardio quando comparado a países de inverno mais rigoroso, nos quais o deslocamento ocorre de forma antecipada e concentrada (BERNARD et al., 2003). Nessa safra agrícola, o primeiro registro de $\boldsymbol{C}$. vitis nas folhas ocorreu em 24 de janeiro na posição mediana, enquanto em 8 de fevereiro foi verificado em folhas de todas as posições. Nos brotos, o primeiro registro ocorreu em 21 de fevereiro (Figura 1A). O pico populacional de $\boldsymbol{C}$. vitis nas folhas medianas e apicais ocorreu em 6 de março, quando foram encontrados, respectivamente, 0,34 e 0,24 espécimes por $\mathrm{cm}^{2}$ (Figura 1A). Nas folhas basais e nos brotos, o pico populacional ocorreu em 19 de março, quando foram encontrados, respectivamente, 0,28 indivíduos por $\mathrm{cm}^{2} \mathrm{e} 0,12$ por broto. Após o pico populacional, verificou-se um intenso declínio da população de $\boldsymbol{C}$. vitis, semelhante ao verificado na Espanha (PÉREZ-MORENO \& MORAZA, 1996) e na Itália (DE LILLO et al., 2004), sem apresentar associação com uso de agrotóxicos, pois, durante o monitoramento, a única aplicação de agrotóxico com reconhecida atividade acaricida foi Kumulus DF em 30 de novembro de 2007 (Figura 1A). Além desse acaricida, foram utilizados, em ambas as safras no período de monitoramento, os seguintes fungicidas e respectivas dosagens: cimoxanil $\left(103,5 \mathrm{~g} \mathrm{ha}^{-1}\right)+$ mancozebe $\left(1.207,5 \mathrm{~g} \mathrm{ha}^{-1}\right)$, cimoxanil $\left(104,3 \mathrm{~g} \mathrm{ha}^{-1}\right)+$ clorotalonil $\left(782,2 \mathrm{~g} \mathrm{ha}^{-1}\right)$, difenoconazol $\left(40,4 \mathrm{~g} \mathrm{ha}^{-1}\right)$, ditianona $(135 \mathrm{~g}$ ha $\left.^{-1}\right)$, hidróxido de cobre $\left(1.451,9 \mathrm{~g} \mathrm{ha}^{-1}\right)$ e iprodiona $\left(359,6 \mathrm{~g} \mathrm{ha}^{-1}\right)$. O movimento descendente de $\boldsymbol{C}$. vitis foi constatado a partir de 21 de fevereiro, quando $15 \%$ dos indivíduos capturados nas armadilhas foram encontrados no sentido ramo-tronco. Em 19 de março, esse comportamento ocorreu em $21 \%$ dos indivíduos e, em 30 de abril, em 27\%. Essa atividade sugere a tendência à hibernação e uma das causas de redução de $\boldsymbol{C}$. vitis nas folhas e armadilhas após o pico populacional, o que encontra respaldo em LOCH (2007), segundo o qual, na Austrália, do início de fevereiro a meados de março, $\boldsymbol{C}$. vitis passa a deslocar-se para os abrigos de hibernação, nas gemas e, principalmente, sob o córtex dos ramos mais espessos e no tronco.

Foi verificado que em todas as datas em que foi realizado o monitoramento simultâneo nas armadilhas adesivas e folhas, o número de $\boldsymbol{C}$. vitis nas armadilhas adesivas (Figura 1A) foi maior que o somatório de indivíduos na área amostrada das folhas. 


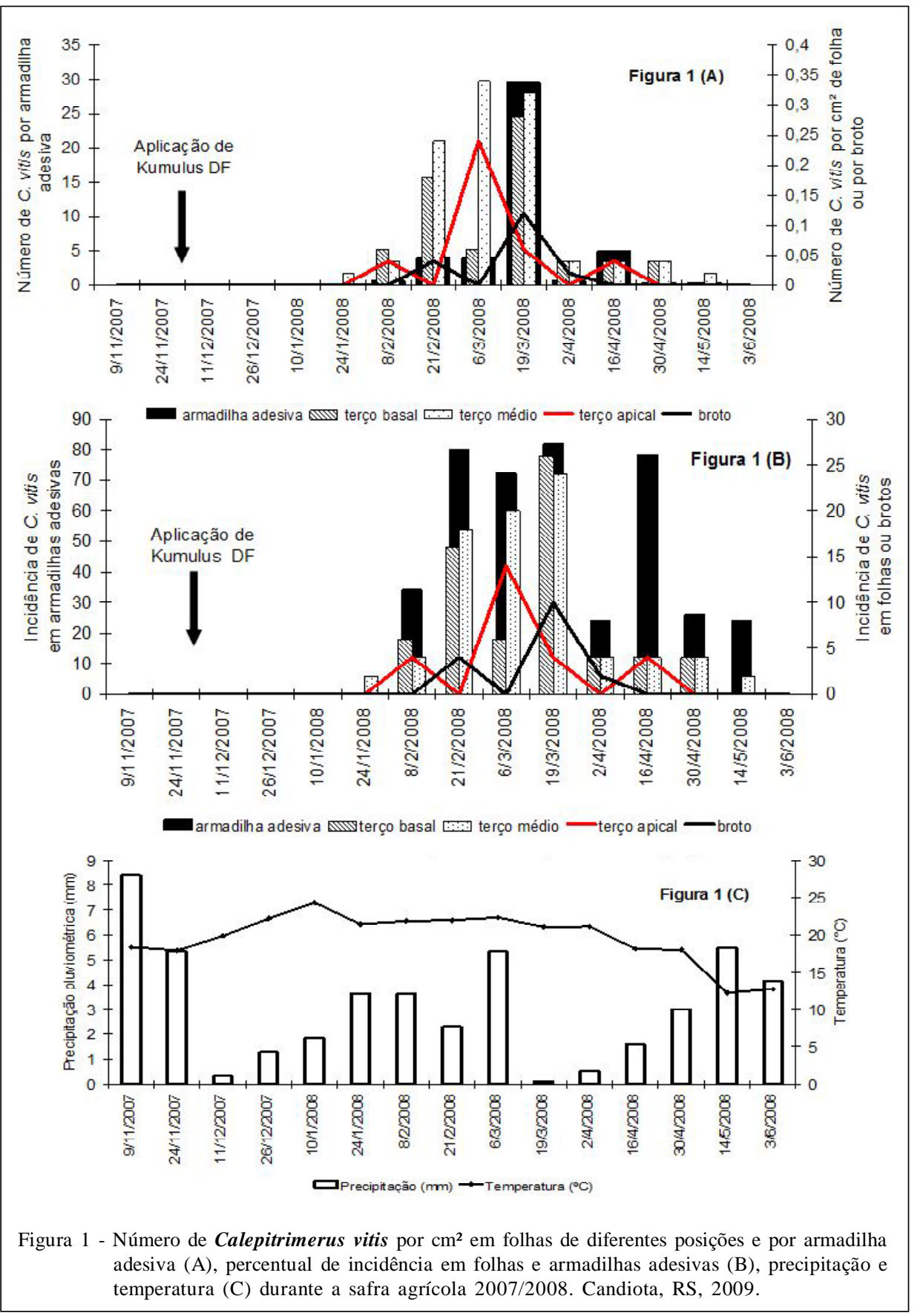

Além disso, o percentual de incidência de $\boldsymbol{C}$. vitis nas armadilhas superou, em todas as oportunidades, o somatório do percentual de incidência nas folhas (Figura 1B). A superioridade das armadilhas adesivas na detecção de $\boldsymbol{C}$. vitis vem ao encontro do verificado por BERNARD et al. (2005), que concluíram que armadilhas adesivas interceptam $89,5 \%$ de $\boldsymbol{C}$. vitis durante o deslocamento nos ramos, sendo uma ferramenta válida para estimar a população da espécie.
A incidência de $\boldsymbol{C}$. vitis nas folhas apresentou uma correlação positiva, significativa e alta $\left(\mathrm{r}^{2}>0,98\right)$ com o crescimento populacional (Tabela 1) evidenciando que a atividade reprodutiva coincide com a dispersão ativa e com a tendência de distribuição generalizada no vinhedo. Relatos similares foram registrados por PÉREZ-MORENO \& MORAZA (1996), os quais encontraram correlações com $\mathrm{r}^{2}=0,92$ entre a incidência e a severidade de $\boldsymbol{C}$. vitis nas folhas. 
Nas armadilhas adesivas, durante o ano agrícola 2007/ 2008, houve uma correlação significativa e menor entre o total de $\boldsymbol{C}$. vitis e a incidência dessa espécie nas armadilhas $\left(\mathrm{r}^{2}=0,42\right)$ (Tabela 1), podendo ser interpretado como um indicativo de eficiência deste método, diante da ilimitada possibilidade de capturas e do limitado aumento da incidência, expressa em porcentagem.

No segundo ano de avaliação, a deteç̧ão de $\boldsymbol{C}$. vitis nas folhas medianas deu-se partir de 2 de outubro de 2008 (Figura 2A) e o pico populacional nessas folhas ocorreu em 30 de outubro de 2008, sendo a densidade de espécimes durante o pico populacional $\left(0,12\right.$ indivíduos por $\left.\mathrm{cm}^{2}\right)$ três vezes menor em relação à safra 2007/2008, quando foram encontrados 0,34 indivíduos por $\mathrm{cm}^{2}$. Nas armadilhas adesivas, semelhante ao verificado nas folhas medianas, as capturas de $\boldsymbol{C}$. vitis iniciaram em 2 de outubro, enquanto o pico populacional ocorreu em 30 de outubro de 2008 (Figura 2A), caracterizando uma antecipação em relação ao ano agrícola anterior para a primeira captura nas armadilhas e em relação ao pico populacional. No primeiro ano de experimentação, durante o pico populacional, foram encontrados 29,5 exemplares de C. vitis por armadilha, já no segundo ano tal valor foi reduzido drasticamente para 0,43 . Acentuadas variações na população de $\boldsymbol{C}$. vitis entre os anos também foram verificadas na Itália, tanto em vinhedos tratados com acaricidas como naqueles sem o controle deste ácaro (DE LILLO et al., 2004) e na Checoeslováquia (HLUCHÝ \& POSPÍŠIL, 1992). No ano agrícola 2008/2009, verificou-se novamente uma correlação significativa e alta entre o número de folhas com incidência de $\boldsymbol{C}$. vitis e a intensidade de infestação nas folhas $\left(r^{2}=0,95\right)$ e entre a incidência nas armadilhas e número de indivíduos capturados $\left(\mathrm{r}^{2}=0,78\right)$ (Tabela 1). Foi observada uma redução acentuada de $\boldsymbol{C}$. vitis nas folhas e nas armadilhas em 27 de novembro de 2008, uma semana após a aplicação de enxofre e, a seguir, novo incremento populacional (Figura 2A), o que se atribui à ação do enxofre se dar pela liberação de compostos gasosos, com curto período de ação (CARMONA \& DIAS, 1996).

De acordo com DUSO et al. (2009), o incremento de calor durante o verão apresenta uma correlação positiva com a intensidade da infestação no ano seguinte, devido ao maior número de gerações de deutóginas hibernantes que se deslocarão para a folhagem na primavera. A maior infestação ocorreu na safra agrícola 2007/2008 com temperatura média mais alta $\left(20^{\circ} \mathrm{C}\right)$, enquanto na safra agrícola $2008 / 2009$, para o mesmo intervalo de avaliação meteorológica, ou seja, 19 de novembro a 3 de junho, a temperatura média foi de $19^{\circ} \mathrm{C}$ (Figuras $1 \mathrm{C}$ e $2 \mathrm{C}$ ), não sendo encontradas,

Tabela 1 - Equações de regressão, coeficientes de determinação $\left(\mathrm{r}^{2}\right)$ e probabilidade (p) dos parâmetros incidência e total de indivíduos de Calepitrimerus vitis em videira durante as safras agrícolas 2007/2008 e 2008/2009. Candiota-RS, 2009.

\begin{tabular}{|c|c|c|c|c|}
\hline Variável Dependente & Variável Independente & Equação de regressão & $\mathrm{r}^{2}$ & $\mathrm{p}$ \\
\hline \multicolumn{5}{|c|}{ 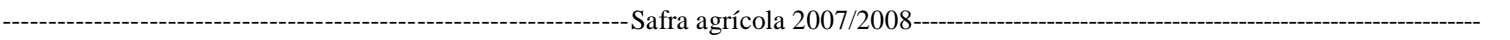 } \\
\hline Incidência $C$. vitis - base & Total $C$. vitis - base & $Y=0,1130+1,8373 x$ & 0,99 & $<0,001$ \\
\hline Incidência $C$. vitis - meio & Total C. vitis - meio & $Y=0,5665+1,3364 x$ & 0,98 & $<0,001$ \\
\hline Incidência $C$. vitis - ápice & Total $C$. vitis - ápice & $Y=0,2249+1,1908 x$ & 0,98 & $<0,001$ \\
\hline Incidência $\boldsymbol{C}$. vitis - broto & Total $\boldsymbol{C}$. vitis - broto & $Y=0,0487+1,6966 x$ & 0,99 & $<0,001$ \\
\hline Incidência $\boldsymbol{C}$. vitis - arm. ${ }^{1}$ & Total C. vitis - arm. & $Y=17,9864+0,0547 x$ & 0,42 & 0,001 \\
\hline Incidência $\boldsymbol{C}$. vitis - arm. & Incidência $C$. vitis - base & $Y=10,3768+3,6113 x$ & 0,64 & $<0,001$ \\
\hline Incidência $\boldsymbol{C}$. vitis - arm. & Incidência $C$. vitis - meio & $Y=7,0323+3,5185 x$ & 0,73 & $<0,001$ \\
\hline Incidência $C$. vitis - arm. & Incidência $C$. vitis - ápice & $Y=16,6032+5,5751 x$ & 0,40 & 0,001 \\
\hline Incidência $\boldsymbol{C}$. vitis - arm. & Incidência $C$. vitis - broto & $Y=18,4352+7,3420 x$ & 0,36 & 0,002 \\
\hline Total C. vitis - arm. & Total $\boldsymbol{C}$. vitis - base & $Y=-49,032+85,8994 x$ & 0,76 & $<0,001$ \\
\hline Total C. vitis - arm. & Total $\boldsymbol{C}$. vitis - meio & $Y=-13,143+44,8753 x$ & 0,46 & 0,007 \\
\hline Total C. vitis - arm. & Total $\boldsymbol{C}$. vitis - ápice & $Y=108,605+33,7858 x$ & 0,07 & 0,239 \\
\hline Total C. vitis - arm. & Total C. vitis - broto & $Y=10,8989+234,169 x$ & 0,89 & $<0,001$ \\
\hline Incidência $\boldsymbol{C}$. vitis - meio & Total $\boldsymbol{C}$. vitis - meio & $Y=-0,1380+0,2674 x$ & 0,95 & $<0,001$ \\
\hline Incidência $\boldsymbol{C}$. vitis - arm. & Total C. vitis - arm. & $\mathrm{Y}=2,1494+0,0954 \mathrm{x}$ & 0,78 & $<0,001$ \\
\hline Incidência $\boldsymbol{C}$. vitis - arm. & Incidência $\boldsymbol{C}$. vitis - meio & $Y=5,5814+0,5610 x$ & 0,19 & 0,006 \\
\hline Total C. vitis - arm. & Total $\boldsymbol{C}$. vitis - meio & $Y=29,2187+2,9963 x$ & 0,54 & 0,002 \\
\hline
\end{tabular}

${ }^{1}$ arm $=$ armadilhas adesivas 


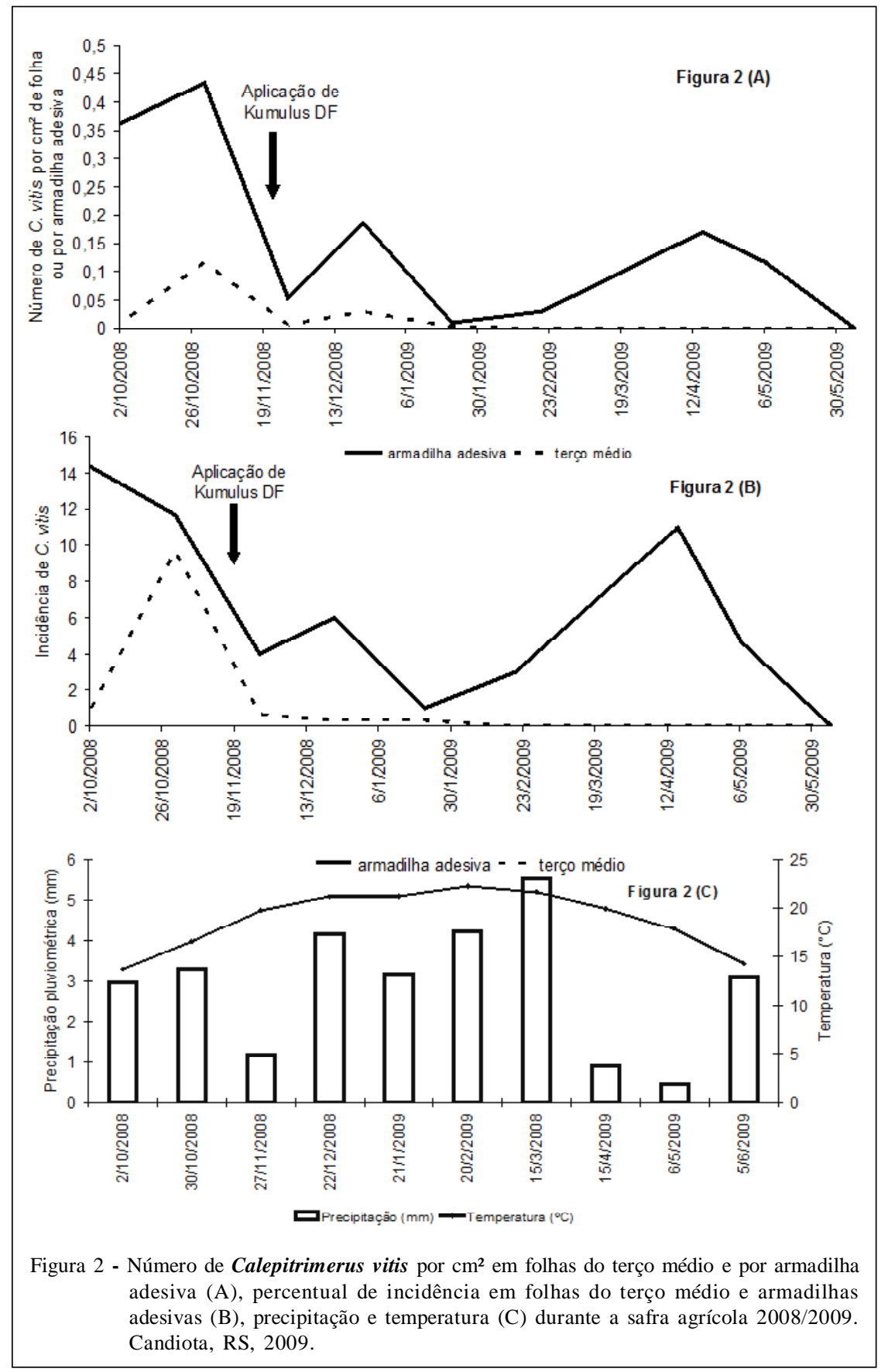

todavia, correlações significativas entre o número de indivíduos nas folhas e nas armadilhas adesivas e a temperatura média observada aos 7 e 14 dias antes das amostragens.

O pico populacional de $\boldsymbol{C}$. vitis, na safra agrícola 2007/2008, coincidiu com o período de menor precipitação pluviométrica (Figura 1C). Tal fato encontra respaldo em PÉREZ-MORENO \& MORAZAZORRILLA (1998) e DE LILLO et al. (2004), para os quais a redução das chuvas favorece o incremento de C. vitis, e também em VANLEEUWEN et al. (2010), que consideram que os ácaros causadores de bronzeamento apresentam incremento populacional em plantas com deficiência hídrica. Não foram encontradas, entretanto, correlações significativas entre o número de indivíduos nas folhas e nas armadilhas com a precipitação média diária aos 7 e 14 dias antes das amostragens nos dois anos de avaliação. 


\section{CONCLUSÃO}

Os níveis populacionais $\boldsymbol{C}$. vitis em videira da cultivar Cabernet Sauvignon em Candiota, na Região da Campanha do Rio Grande do Sul, apresentam diferenças de intensidade entre as safras agrícolas. Há alta correlação entre o número de $\boldsymbol{C}$. vitis por $\mathrm{cm}^{2}$ na face abaxial das folhas de videira e o percentual de folhas com incidência desse ácaro. As armadilhas adesivas são mais eficientes na detecção de $\boldsymbol{C}$. vitis nos ramos de produção em relação à avaliação direta na face abaxial das folhas de videira.

\section{REFERÊNCIAS}

BERNARD, M. et al. Movement, distribution and effects of rust mite (Calepitrimerus vitis) and preliminary studies of bud mite (Colomerus vitis) on grapevines. In: EMMETT, B. (Org.). Strategic use of sulphur in integrated pest and disease management (IPM) programs for grapevines. Victoria: Department of Primary Industries/Primary Industries and Resources and Resources South Australia/Centre for Environmental Stress and Adaptation Research/La Trobe University, 2003. p.133-152. Disponível em: <http:// www.sardi.sa.gov.au/__data/assets/pdf_file/0018/46620/ sulphur_report.pdf >. Acesso em: 18 jan. 2010.

BERNARD, M. et al. Eriophyoid mite damage in Vitis vinifera (grapevine) in Australia: Calepitrimerus vitis and Colomerus vitis (Acari: Eriophyidae) as the common cause of de widespread 'Restricted Spring Growth' syndrome. Experimental and Applied Acaralogy, Amsterdam, v.35, n.1, p.83-109, 2005. Disponível em: <http://www.springerlink.com/content/ m7318104372h4018/>. Acesso em 20: jun. 2009. doi: 10.1007/ s10493-004-1986-4.

CARMONA, M.M.; DIAS, J.C.S. Fundamentos de acarologia agrícola. Lisboa: Fundação Calouste Gulbenkian, 1996. 423p.

DE LILLO, E. et al. Efficacy of fungicides and acaricides against Calepitrimerus vitis (Nalepa). Phytophaga. Amsterdam, v.14, p.599-603, 2004.

DUSO, C. et al. Pollen availability and abundance of predatory phytoseiid mites on natural and secondary hedgerows. BioControl, Dordrecht, v.49, n.4, p.397-415, 2004. Disponível em: <http:// www.springerlink.com/content/k422314528136382/>. Acesso em: 11 abr. 2010. doi:10.1023/B:BICO.0000034601.95956.89.

DUSO, C. et al. The impact the eriophyoids on crops: recent issues on Aculus schlechtendali, Calepitrimerus vitis and Aculops lycopersici. Experimental and Applied Acarology, Amsterdam, v.51, p.1-14, 2009. Disponível em: <http:// www.springerlink.com/content/580pl27150725662/>. Acesso em: 19 mar. 2010. doi: 10.1007/s10493-009-9300-0.

HLUCHÝ, M.; POSPÍŠIL, Z. Damage and economic injury levels of eriophyid and tetranychid mites on grapes in Czechoslovakia. Experimental and Applied Acarology, Amsterdam, v.14, p.95-106, 1992. Disponível em: <http:// www.springerlink.com/content/g0530228573102t2/>. Acesso em: 10 mar. 2010. doi: 10.1007/BF01219102.
JAMES, D.G.; WHITNEY, J. Mite populations on grapevines in south-eastern Australia: implications for biological control of grapevines mites (Acarina: Tenuipalpidae, Eriophyidae). Experimental and Applied Acarology, Amsterdam, v.17, p.259-270, 1993. Disponível em: <http://www.springerlink.com/ content/b677554600709730/>. Acesso em: 10 mar. 2010. doi: 10.1007/BF02337275.

KEIFER, H.H. Eriophyid studies XII. California: Department of Agriculture, 1942. 13p. (Bulletin, v.31, n.3).

KLOCK, C.L. Bioecologia de ácaros em videira (Vitis vinifera L.: Vitaceae) no Rio Grande do Sul, Brasil. 2008. 101f. Dissertação (Mestrado em Ambiente e Desenvolvimento) - Curso de Pós-graduação em Ambiente e Desenvolvimento, Centro Universitário UNIVATES, RS.

LOCH, A. Grapevine pests and their management. Wagga Wagga: NSW Department of Primary Industries, 2007. 8p. (Primefact, 511). Disponível em: <http://www.dpi.nsw.gov.au/ data/assets/pdf_file/0010/110998/grapevine-pests-and-theirmanagement.pdf $>$. Online. Acesso em: 28 abr. 2010.

MONFREDA, R. et al. Collection and detection of eriophyoid mites. Experimental and Applied Acarology, Amsterdam, v.51, n.1-3, p.273-282, 2010. Disponível em: <http:// www.springerlink.com/content/p7651527821u03 $7 />$ Acesso em: 15 abr. 2010. doi: 10.1007/s10493-009-9315-6.

MORAES, G.J.; FleChtMANN, C.H.W. Manual de acarologia: acarologia básica e ácaros de plantas cultivadas no Brasil. Ribeirão Preto: Holos, 2008. 308p.

PÉREZ-MORENO, I.P.; MORAZA, M.L. Método de muestro secuencial-enumerativo y binomial para Calepitrimerus vitis (Nalepa, 1905) (Acari: Eriophyidae). Boletín Sanidad Vegetal Plagas, Madrid, n.22, p.179-187, 1996. Disponível em: <http://www.mapa.es/ministerio/pags/biblioteca/plagas/ BSVP-22-01-179-187.pdf $>$. Acesso em: 19 maio, 2010.

PÉREZ-MORENO, I.P.; MORAZA-ZORRILLA, M.L. Population dynamics and hibernation shelters of Calepitrimerus vitis in the vineyards of Rioja, Spain, with a description of a new eriophyid extraction technique (Acari: Eriophyidae). Experimental and Applied Acarology, Amsterdam, v.22, p.215-226, 1998. Disponível em: <http://www.springerlink.com/ content/g35k441511p21h34/>. Acesso em: 18 abr. 2010. doi: 10.1023/A:1006014331156.

STRECK, E.V. et al. Solos do Rio Grande do Sul. 2.ed. Porto Alegre: EMATER/RS, 2008. 220p.

VAN LEEUWEN, T. et al. The control of eriophyoid mites: state of art and future challenges. Experimental and Applied Acarology, Amsterdam, v.51, n.1-3, p.205-224, 2010. Disponível em: <http://www.springerlink.com/content/br1795172h656m53/>. Acesso em: 8 abr. 2010. doi: 10.1007/s10493-009-9312-9.

WALTON, V.M. et al. Relationship between rust mites Calepitrimerus vitis (Nalepa), bud mites Colomerus vitis (Pagenstecher) (Acari: Eriophyidae) and short shoot syndrome in Oregon vineyards. International Journal Acarology, Abingdon, v.33, n.4, p.307-318, 2007. Disponível em: <http:/ /entomology.tfrec.wsu.edu/VPJ_Lab/downloads/U_Chambers/ 07-Walton-VP\%2833-4\%29.pdf $>$. Acesso em: 15 mar. 2010. doi: $10.1080 / 01647950708683691$. 